\title{
Verzeichnis der bis jetzt bekannten Arten der Dipterengattung Drapetis Meigen.
}

\author{
Von Prof. M. Bezzi, Sondrio, Italien.
}

In meiner Arbeit über die indoaustralischen Empididen, gesammelt von Herrn L. Biró (Annales Musei nationalis hungarici II, 320-361, 1904) habe ich die Gattung Drapetis in zwei Untergattungen (Drapetis s. str. und Ctenodrapetis) geteilt; leider ist es unmöglich, die bis jetzt bekannt gewordenen Arten nach den Beschreibungen in diesen zwei Abteilungen zu ordnen. Die Arten scheinen in tropischen Gegenden besonders häufig zu sein, sind aber sehr klein und daher leicht zu übersehen; nichtsdestoweniger ist eine ziemlich große Anzahl von Arten bekannt, wie aus folgendem Verzeichnis zu ersehen ist.

Eine ausführlichere Synonymie der paläarktischen Arten ist im zweiten Bande des Kataloges der paläarktischen Dipteren, p. 272-274, zu finden; diese Arten sind hier ohne Citate angeführt. aenea Walk., Kat. 272. - Africa sept.

aenescens Wied., Kat. 272. — Africa, Asia, Europa.

brunnipes Macq.

crassa Loew.

affinis Lw. = assimilis Fall. ambigua Macq. = flavipes Macq.

apicis Will., Trans. ent. Soc. London 1896, 442, 3. T, XIV, Fig. 167 (1896); Mel., Trans. am. ent. Soc. XXVIII. 209 (1902). - America centr.

arcuata Loew, Kat. 272 . Europa.

minima Zett. nec Meig.

assimilis Fall., Kat. 272. - Europa.

affinis Lw.

minima Meig.

nigra Meig.

pygmaea v. Ros.

aterrima Curt., Kat. 273. - Europa.

atra Walk.

bihamata Bezzi, Ann. Mus. nat. hung. ПI, 353, 35 (1904). N.-Guinea.

brumipes Hacq. = aenescens Wied.

callosotibia Bezzi, Annal. Mus. nat. hung. II. 354, 37 (1904). N.-Guinea. 
ciliatocosta Bezzi, Ann. Mus. nat. hung. П., 355, 39, Fig. 6 (1904). - Australia.

crassa Lw. = aenescens Wied.

curvinervis Zett. = moriella Zett.

curvipes Meig. = flavipes Macq.

disco oidalis Bezzi, Ann. Mus. nat. hung. П, 355, 38 (1904). - India. distans nom. nov. - N.-Guinea.

divergens Bezzi (nec Loew), Ann. Mus. nat. hung. II, 353, 34 (1904). divergens Bezzi $=$ distans nom. nov.

divergens Loew, Berl. ent. Zeitschr. XVI. 90, 62 (1872); Mel., Trans. am. ent. Soc. XXVIII. 210, T. V, Fig. 15 (1902). America sept.

mimuta Will., Trans. ent. Soc. London. 1896, 442, 4. T. XIV. Fig. 168(1896); Mel.,Trans.am. ent. Soc.XXVIII 210 (1902). dividua Mel., Trans. am. ent. Soc. XXVIII, 208, T. V. Fig. 16, 18, 23 (1902). - America sept.

exilis Meig., Kat. 273. - Europa.

exilis Zett. = pusilla Lw.

fascipes v. Ros. = flavipes Macq.

femoralis Wheel. et Mel., Biolog. centr. amer. I. 376.1 (1901);

Mel., Trans. am. ent. Soc. XXVIII. 211, T. V, Fig. 13 (1902).

America centr.

flavipes Macq., Kat. 273. - Europa.

ambigua Macq.

curvipes Meig.

exilis Macq. p. p.

fascipes v. Ros.

nigra Fll.

flavipes Will. = gilvipes Lw.

flexuosa Loew = moriella Zett.

fulvida Will. = Tachista eadem.

gilvipes Loew, Berl. ent. Zeitschr. XVI. 89, 61 (1872); Mel., Trans. am. ent. Soc. XXVIII. 213 et 340 (1902). America sept.

flavipes Will. (nec Macq.) Trans. ent. Soc. London 1896.441 (1896). xanthopoda Will., Trans. ent. Soc. London 1896, 308, 1, T. XI, Fig. 85 (1896); Mel., Trans. am. ent. Soc. XXVII, 212 (1902).

gracilis Bezzi, Ann. Mus. nat. hung. II., 354, 37 (1904). N.-Guinea. 
Iatipennis Mel., Trans. am. ent. Soc. XXVIII. 209. (1902). T. V. F. 11, 19. - America sept.

Iuteipes 01., Kat. 273. - Europa.

marginata Meig., Kat. 273. -- Europa.

medetera Mel. = Elaphropeza eadem.

minima Meig. = assimilis Fall.

minima Zett. = arcuata $\mathrm{Lw}$.

minuta Will. = divergens $\mathrm{Lw}$.

moriella Zett., Kat. 273. - Europa.

curvinervis Zett.

flexuosa Lw.

nervosa Lw. = nigritella Zett.

nigra (Fall.?) Zett. = flavipes Nacq.

nigra Meig. = assimilis Fall.

nigripes Zett., Kat. 274. - Europa.

nigritella Zett., Kat. 274. - Europa.

nervosa Lw.

nitida Mel. = unipila Lw.

obscuripennis Bezzi = phaeoptera nom. nov.

obscuripennis Phil., Verh. zool. bot. Ges. Wien XV. 768,

2 (1865). - America mer.

phaeoptera nom. nov. - N.-Guinea.

obscuripennis Bezzi (nec Phil.), Ann. Mus. nat. hung. II. 351.

32 (1904).

pilipes Loew, Kat. 274. - Europa.

pubescens Loew, Berl. ent. Zeitschr. VI. 210, 57 (1862);

Mel., Trans. am. ent. Soc. XXVII. 210 et 340 (1902).

- America sept.

pusilla Loew., Kat. 274. - Europa.

exilis Zett. nec Meig.

pygmaea v. Ros. = assimilis Fall.

rubrithorax Bezzi, Ann. Mus. nat. hung. П. 356, 40 (1904).

- N.-Guinea.

septentrionalis Mel., Trans. am. ent. Soc. XXVIII. 211

(1902). - America sept.

setigera Loew, Kat. 274 . - Europa.

spectabilis Mel., Trans. am. ent. Soc. XXVIII, 212, T. V.

Fig. 9-20 (1902). - America sept.

unipila Loew, Berl. ent. Zeitschr. XVI. 88, 60 (1872); Mel., Trans. am. ent. Soc. XXVIII. 211 et 339 (1902). - America sept. 
nitidd Mel., Trans. am. ent. Soc. XXVIII. 207, T. V. Fig. 10, 14, 21 (1902).

valdiviana Phil., Verh. zool. bot. Ges. Wien XV. 768, 1 (1865). - America mer.

xanthopoda Will. = gilvipes $\mathrm{Lw}$.

xanthopoda Will. 1896 , p. $441=$ Tachista fulvida (Will.) Coquill.

xanthopyga Bezzi, Ann. Mus. nat. hung. ПI. 352, 33 (1904). - N.-Guinea.

Es sind im ganzen 36 Arten bekannt, von denen wahrscheinlich aenea Walk. = aenescens Wied. und luteipes $01 .=$ flavipes Macq. sind. Von diesen 36 Arten gehören 14 dem paläarktischen Faunengebiete an, 10 dem nearktischen, 2 dem neotropischen, 1 dem südasiatischen, 8 dem australischen; eine Art (aenescens Wied.) scheint in Europa und Afrika (und Asien) weit verbreitet zu sein.

\section{Abbildungen von Grottenkäfern aus Bosnien und der Herzegowina.}

Von Edm. Reitter in Paskau (Mähren).

Hiezu Tafel I., Fig. 1 bis 9.

Erklärung der Figuren:

1. Antroherpon Leonhardi Reitt. Wiener Ent. Zeitg. 1902, 208. (Herzegowina).

2. Antroherpon Matuliči Reitt. Wien. Ent.Ztg. 1903,216.(Herzegowina).

3. Antroherpon Loreli Zoufal, Wien. Ent. Ztg. 1904. 20, vom 1. Februar.

= A. Kraussi Müll., Münch. Kol. Zeitschr. 1904. (II.) pg. 38, vom 16. Februar.

4. Pholeuonopsis Sequensi Reitt. Wien. Ent. Ztg. 1902. 223, aus dem nördlichen Teile von Bosnien.

5. Anillocharis Ottonis Reitt. Wien. Ent.Ztg. 1903. 231. (Herzegowina).

6. Leonhardella angulicollis Reitt. Wien. Ent. Ztg. 1903. 209. (Herzegowina).

7. Silphanillus Leonhardi Reitt. Wien. Ent. Ztg. 1903. 211. Herzegowina. Unter einem großen, tief in der Erde eingebetteten Steine an der höchsten Stelle der Vlasulja entdeckt.

8. Bathyscia (Aphaobius) eurycnemis Reitt. Wien. Ent. Ztg. 1904. 26. (Herzegowina).

9. Anophthalmus Hilf $i$ Reitt. Wien. Ent.Ztg. 1903. 212. (Herzegowina). 


\section{$2 \mathrm{BHL}$ Biodiversity Heritage Library}

Bezzi, Mario. 1904. "Verzeichnis der bis jetzt bekannten Arten der

Dipterengattung Drapetis MEIGEN." Wiener entomologische Zeitung 23, 143-146. https://doi.org/10.5962/bhl.part.27198.

View This Item Online: https://www.biodiversitylibrary.org/item/44102

DOI: https://doi.org/10.5962/bhl.part.27198

Permalink: https://www.biodiversitylibrary.org/partpdf/27198

\section{Holding Institution}

Smithsonian Libraries

\section{Sponsored by}

Smithsonian

\section{Copyright \& Reuse}

Copyright Status: NOT_IN_COPYRIGHT

This document was created from content at the Biodiversity Heritage Library, the world's largest open access digital library for biodiversity literature and archives. Visit BHL at https://www.biodiversitylibrary.org. 